\title{
Phasen, Zustandswechsel und die einfache Phasensemantik
}

\author{
VOLKMAR ENGERER
}

Staatsbibliothek Aarhus, Dänemark

\begin{abstract}
Taking a preliminary periodization of phasal verb research as a starting point, the article argues for an implicit connection between four historical phases of research and five features of grammatical description of phasal verbs. The remainder of the contribution focuses on one period, in particular the formal semantic approaches inspired by Montague grammar in the late 1970s, with David Dowty as the perhaps most prominent figure. The article shows that early formal semantics is characterised by interpreting the most basic feature in phasal verb grammar, Simple Phasal Semantics (SPS), as a change-of-state concept. This move establishes phasal meaning as a basic feature in lexicon structure, and, in consequence, characterises phasal verbs as lexicalisations of overall semantic features. To substantiate this analysis, the essentials of SPS are presented, followed by a discussion of Dowty's conception of change-of-state in his reanalysis of Vendler's four aspectual verb classes in English.
\end{abstract}

\section{Historische Phasen der Phasenverbforschung}

Obwohl „Phasenverben“, eine dreigeteilte Gruppe quasi-grammatikalischer Verben bestehend aus beginnen, aufhören und fortsetzen, beileibe kein etablierter Terminus in der Linguistik (und erstrecht nicht in der germanistischen Linguistik) ist, spielt das entsprechende Konzept - auf das ich noch eingehen werde - eine mitunter vielleicht unscheinbare, aber bei genauerem Hinsehen äußerst interessante Rolle in der linguistischen Forschung der vergangen 50 Jahre. Über ihre Funktion als Testeinheiten hinaus - meist auf Grund ihrer spezifischen Selektionsbeschränkungen in Verbindung mit aktionsartmarkierten Syntagmen - wurden (und werden) Phasenverben in der Forschung als grammatische Gegenstände in eigenem Recht betrachtet. Ich möchte einleitend eine erste Periodisierung der linguistischen Erforschung von Phasenverben ca. ab 1960 vorschlagen, und einen historischen, inhaltlich (und

Tidsskrift for Sprogforskning, årgang 6, 2008 Århus: Statsbiblioteket

Artikel nr. 5, Engerer, Volkmar, 17 pp.

http://ojs.statsbiblioteket.dk/index.php/tfs/index 
grammatiktheoretisch) bedingten Zusammenhang zwischen den chronologisch aufeinanderfolgenden Phasen ihrer Erforschung motivieren.

Von der frühen Transformationsgrammatik der 60er Jahre und ihren Nachwirkungen in den 70er Jahren, wo vor allem syntaktische Eigenschaften von Phasenverben wie Anhebung („Raising“) oder Kontrolle im Vordergrund standen (Phase I), ist die Gruppe formalsemantisch orientierter Ansätze zu nennen, die exemplarisch von David Dowty gegen Ende der 70er Jahre vertreten worden sind (vgl. Dowty 1979) (Phase II). Letztere machen den Schritt zur Hypostasierung des Phasenkonzepts als Zustandswechsel, ein Schachzug, der die Phasensemantik als generellen Zug im Lexikon verabsolutiert und damit Phasenverben als Lexikalisierungen allgegenwärtiger semantischer Merkmale auszeichnet. Auf diese Phase werde ich mich in diesem Beitrag konzentrieren.

Als weitere Periode neben der transformationsgrammatischen Syntaktisierung in der Anfangszeit (I) und der formalsemantischen Verabsolutierung von Phasenverben in den 70er Jahren (II) sind die synthetischeren grammatiktheoretischen Ansätze der Beschreibung von Phasenverben der 80er, Anfang der 90er Jahre hervorzuheben, wie sie z.B. in Jackendoffs „Conceptual semantics" und Theorie kognitiver Strukturen vorliegen (Phase III). Jackendoffs Erkenntnisse bereiten den Grund für die nächste und vorläufig letzte Phase der Erforschung von Phasenverben, nämlich die exakte Beschreibung der syntagmatischen Prozesse, welche, ausgehend von Phasenverbkonstituenten, sich auf die syntaktischen Nachbarkonstituenten von Phasenverben ausweiten (vgl. z.B. die erzwungene Uminterpretation in Die Schule beginnt von GEBÄUDE zu SCHULTAG/SCHULJAHR, oder die Interpolation von ,lesen', ,schreiben', aber nicht *'zerreißen', usw. in Peter beginnt ein Buch) (letztere übrigens schon erkannt in Newmeyer 1975). Als ein hervorragendes Beispiel für Analysen in Phase IV in den 90er Jahren ist Pustejovskys Theorie des generativen Lexikons hervorzuheben (Pustejovsky 1995), welche zum ersten Mal diese mehr dynamischen Problematiken einer Phasenverbgrammatik unter die Lupe nimmt.

Neuere Tendenzen in der linguistischen Phasenverbforschung sind meines Erachtens in der Sprachtypologie zu verorten, wo sprachübergreifend ähnliche grammatische Züge von Phasenverben (und Phasenbedeutungen) in genetisch nicht verwandten Sprachen herausgearbeitet werden. Zum jetzigen Zeitpunkt ist 


\section{PHASEN, ZUSTANDSWECHSEL UND DIE EINFACHE PHASENSEMANTIK}

darüber jedoch nur schwer etwas Gesichertes zu sagen. Ich möchte also, von der relativ sicheren Warte von 2008 aus gesehen, die folgenden vier forschungshistorischen Ansätze unterscheiden:

1. Frühe Transformationsgrammatik (60er, Anfang 70er Jahre) Syntaktisierung von Phasenverben

2. Formale (modelltheoretische) Semantik (70er, Anfang der 80er Jahre) Semantisierung von Phasenverben

3. Konzeptuelle Ansätze (80er, Anfang der 90er Jahre) - Synthese von Semantik und Syntax von Phasenverben

4. Theorien des Generativen Lexikons (90er Jahre) - Syntagmatische Dynamisierung von Phasenverben

Diese Periodisierung spiegelt, wie ich nun zeigen will, einen grammatischen Beschreibungsbedarf, der zur grammatischen Vollständigkeit, genauer: Adäquatheit, strebt, wider. Es kann meiner Meinung nach nämlich gezeigt werden, dass die Phasen (I) bis (IV) nicht willkürliche Aspekte der Phasenverbgrammatik aufgreifen, sondern historisch mehr oder weniger "gezielt" (wenn diese teleologische Metapher im Bereich des Historischen einmal erlaubt ist) einen Bereich nach dem anderen abarbeiten, um schließlich alle theoretisch relevanten Beschreibungsaspekte abzudecken. Ich will kurz demonstrieren, was ich meine, und zu diesem Zweck den grammatischen Bereich abstecken, der meiner Meinung nach als Richtschnur für eine adäquate Beschreibung von Phasenverben dienen kann.

\section{Grammatische Merkmale von Phasenverben}

In Engerer (2008) habe ich fünf grammatische Beschreibungsmerkmale für Phasenverben vorgeschlagen, welche ein System von Abhängigkeiten zwischen grammatischen Eigenschaften entwerfen und die grammatische Beschreibung strukturieren. In Diagrammform sehen die Merkmale und ihre Relationen so aus: 


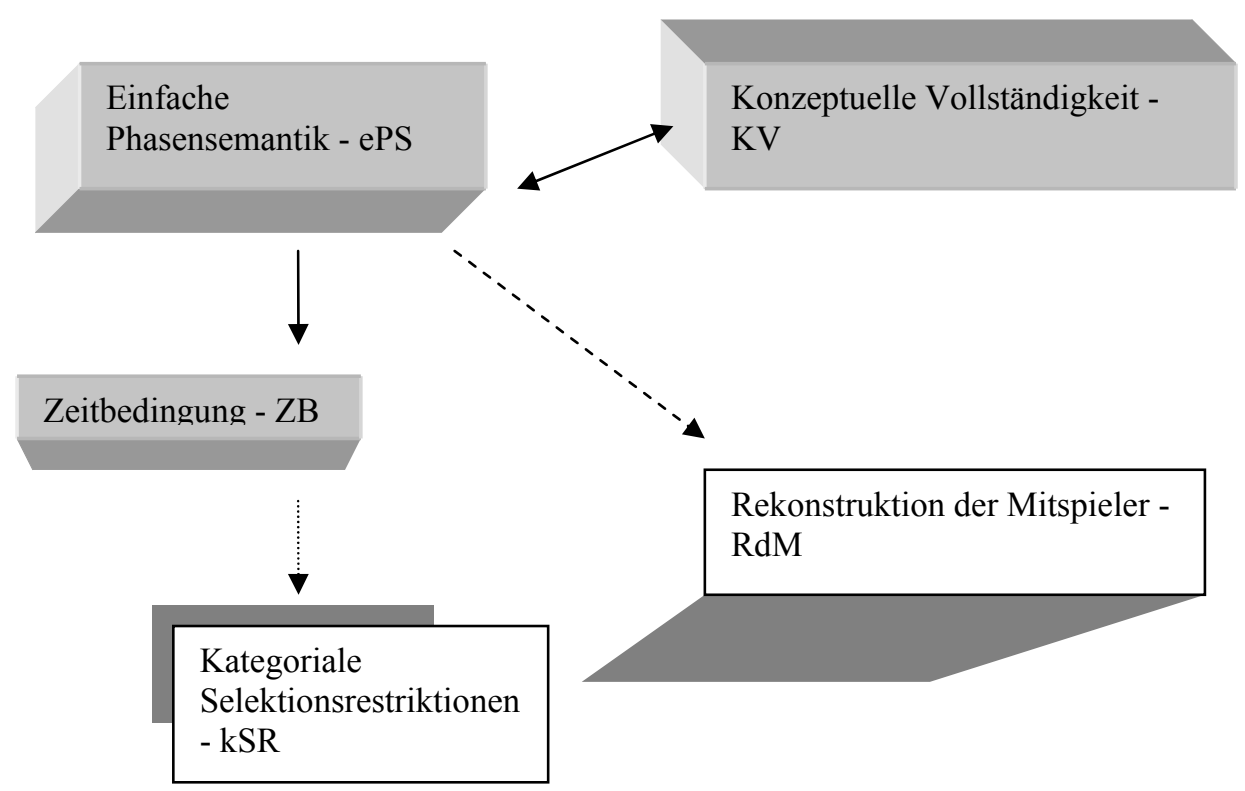

Figur: Grammatische Merkmale von Phasenverben (nach Engerer 2008)

Die Definition der Einfachen Phasensemantik ePS benützt ein kleines Instrumentarium logischer Hilfsmittel (z.B. Implikation, Präsupposition, Negationszeichen usw., vgl. Engerer 2002), welche in einer einfachen Variation die drei Phasen beschreiben. Da ich im Folgenden die ePS noch näher erläutern und problematisieren werde, möchte ich es hier bei diesen kurzen Bemerkungen belassen. ePS hängt mit dem Merkmal der Konzeptuellen Vollständigkeit KV zusammen, das besagt, dass die Phasenverbkategorie in die drei Untergruppen ingressiv, egressiv und kontinuativ zerfällt - und nicht mehr. Die drei Teilphasen erschöpfen sozusagen ein Intervall, und nach der These der KV sollte es keine Sprachen geben, die mehr als drei Phasen konzeptualisieren - oder andere als die drei genannten. Das dritte Merkmal ist die Zeitbedingung ZB, die besagt, dass Phasenverben allen ihren syntaktischen Argumenten ein Minimum von Zeitstruktur auferlegen, egal, ob es sich um Infinitiv- oder nominale Komplemente handelt und ungeachtet, in welcher syntaktischen Position das Argument steht (Subjekte und Objekte sind gleichermaßen betroffen). Wie die Zeitbedingung arbeitet, habe ich in Engerer (2002) gezeigt. Die ZB ist in meiner 


\section{PHASEN, ZUSTANDSWECHSEL UND DIE EINFACHE PHASENSEMANTIK}

Konzeption (vgl. die graphische Darstellung weiter oben) das Bindeglied zwischen ePS und dem syntaktischen Merkmal der Kategorialen Selektionsrestriktionen kSR, welches die speziellen kategorialen Valenzeigenschaften von Phasenverben implementiert. Im Deutschen gilt z.B., dass Phasenverben Infinitiv- und nominale Komplemente nehmen, finite Satzargumente jedoch ausgeschlossen sind. Das fünfte und letzte Merkmal von Phasenverben ist RdM - Rekonstruktion der Mitspieler. Das syntaktische Subjekt von Phasenverben bekommt in vielen Konstruktionen vom Phasenverb keine Theta-Rolle zugewiesen, es ist, dem Theta-Kriterium entsprechend (vgl. Stechow/Sternefeld 1988: 269ff.), der vom Phasenverb eingebettete infinitivische und subjektlose Ausdruck, der das oberflächliche Phasenverb-Subjekt thematisch markiert und auch sonst semantisch restringiert.

\section{FORSCHUNGSGESCHICHTE UND DESKRIPTIVE ADÄQUATHEIT}

Ich meine, und kehre nun von meinem Exkurs in die Grammatik zur Forschungshistorie zurück, dass anhand dieser fünf Merkmale wichtige Beziehungen der Grammatik von Phasenverben zu verschiedenen Phasen ihrer Erforschung, sowie eine historische Tendenz zu Beschreibungsadäquatheit eines grammatischen Teilbereiches identifiziert werden können. Die zeitlich und schulenmäßig so unterschiedlichen Ansätze fokussieren jeweils, wie ich zeigen möchte, auf komplementäre Beschreibungsaspekte der grammatischen Struktur von Phasenverben, so dass der historische Rückblick durchaus Hinweise auf die Adäquatheit eines aktuellen Forschungsdesigns liefern kann. Im Vorgriff auf meine kommenden Analysen möchte ich einige Beispiele solch „historisch motivierter" Hypothesen formulieren.

Die oben genannten Ansätze (I)-(IV) unterscheiden sich übergeordnet, ob sie (einseitig) auf ein bisher unterbelichtetes grammatisches Merkmal fokussieren oder eine Synthese bereits erkannter und analysierter grammatischer Merkmale anstreben. Zum Fokussierungstyp gehören bei erstem Hinsehen (I) und (II), welche im Fall von (I) auf die syntaktischen Eigenschaften (kSR und RdM) und im Fall von (II) auf die Formulierung der adäquaten Semantik (ePS) hinsteuern. (III), konzeptuelle Ansätze, sind in der Form, wie sie von Jackendoff vertreten wird, dem Synthesetyp zuzurechnen, wo die syntaktischen Fokussierungen von (I) mit den semantischen Fokussierungen von (II) in Beziehung gesetzt und in einem zusammenhängenden Beschreibungsmodell eingebaut werden. (IV), 
Theorien des generativen Lexikons, wiederum liefern in Bezug auf die Analyse von Phasenverben hauptsächlich neue Erkenntnisse im Bereich der syntagmaübergreifenden ZB, was (IV) erneut dem Fokussierungstyp nahe bringt.

Dieses historische Abwechseln von Fokussierung und Synthese hat heute dazu geführt, dass vier der fünf Merkmale der grammatischen Beschreibung von Phasenverben, ePS (II), ZB (IV) sowie kSR und RdM (beide I), durch eine historische Phase in der Geschichte der Linguistik abgedeckt sind. Diesen Typ von Entwicklung meinte ich mit „historischer Tendenz zur Beschreibungsadäquatheit“. Das fünfte Merkmal, Konzeptuelle Vollständigkeit, ist explizit in keinem der besprochenen Ansätze erwähnt, obwohl formale sowie kognitiv-konzeptuelle Argumente für das Vollständigkeitspostulat implizit durchaus in den jeweiligen semantischen Konzeptionen von (II) und (III) vorhanden sind. Ich meine aber, ohne es beweisen zu können, dass neuere typologische Untersuchungen von Phasenverben in exotischen und nicht verwandten Sprachen als ein historisch bedingter Versuch gedeutet werden können, konzeptuelle Vollständigkeit als den, nach meiner These, letzten Baustein zur deskriptiven Adäquatheit auf empirische Beine zu stellen. Aber das nur am Rande.

Im Folgenden werde ich mich auf die Phase II konzentrieren, die Gruppe formalsemantisch orientierter Ansätze, die exemplarisch von David Dowty gegen Ende der 70er Jahre vertreten worden sind (vgl. Dowty 1979). Kennzeichnend für sie ist der Schritt zur Hypostasierung der Einfachen Phasensemantik als Zustandswechsel, ein Schachzug, der die Phasensemantik als generellen Zug im Lexikon verabsolutiert und damit Phasenverben als Lexikalisierungen allgegenwärtiger semantischer Merkmale auszeichnet. Ich werde also zunächst die Grundzüge einer Einfachen Phasensemantik darstellen.

\section{EINFACHE PHASENSEMANTIK EPS}

Der Einfachheit halber betrachten wir Verbalphrasen VP, die sich aus einem Phasenverb PV im Präteritum (begann, fuhr fort, hörte auf) und einem Infinitivkomplement K (z.B. zu schreiben) zusammensetzen, die Strukturformel für die Verbalphrase ist also (Engerer 2002: 60):

$$
\mathrm{VP} \rightarrow \mathrm{PV} \mathrm{K}
$$


Für die Darstellung von Sätzen S verwenden wir einen Platzhalter $\mathrm{X}$ an der Subjektstelle mit gleichzeitiger Klammerung und Indizierung der Verbalphrase:

(2) $\mathrm{S} \rightarrow \mathrm{X}[\mathrm{PV} \mathrm{K}]_{\mathrm{VP}}$

Weiterhin benötigen wir eine einfache Subkategorisierung von PV in ingressive, egressive und kontinuative Verben (um die Darstellung zu vereinfachen, wieder im Präteritum):

(3a) $\quad \mathrm{PV}_{\text {ingr }}=\{$ begann, startete, setzte ein, $\ldots\}$

(3b) $\mathrm{PV}_{\mathrm{egr}}=\{$ hörte auf, beendete, schloss $a b, \ldots\}$

(3c) $\quad \mathrm{PV}_{\mathrm{kont}}=\{$ setzte fort, fuhr fort, ... $\}$

Um die Darstellung weiter zu vereinfachen, lassen wir Projektion der Phasenmerkmale ingr, egr und kont von PV auf die höheren Konstituenten VP und $\mathrm{S}$ zu:

(4a) $\quad \mathrm{VP} \rightarrow \mathrm{PV}_{\text {ingr/egr } / \text { kont }} \mathrm{K}={ }_{\text {def }} \mathrm{VP}_{\text {ingr/egr }} /$ kont $\rightarrow$ PV K

(4b) $\mathrm{S} \rightarrow \mathrm{X}[\mathrm{PV} \mathrm{K}]_{\mathrm{VPingr} / \text { egr } / \text { kont }}=\mathrm{def}_{\mathrm{def}} \mathrm{S}_{\text {ingr/egr } / \text { kont }} \rightarrow \mathrm{X}[\mathrm{PV} \mathrm{K}]_{\mathrm{VP}}$

Zuletzt nehmen wir einen Zeitpunkt $t_{e}$ an, der meist als Betrachtzeitpunkt durch das Tempus gegeben ist und an dem die Wahrheit des Satzes mit Phasenverb evaluiert wird. Diese Fassung der ePS, formuliert in Implikations- und Präsuppositionsbeziehungen, folgt einem Vorschlag von Fabricius-Hansen (1975: 87ff.) und kann für die einzelnen Phasenverbgruppen einfach formuliert werden. Ein Satz mit ingressivem Phasenverb $S_{\text {ingr }}$, z.B. Er begann sich auf die Prüfung vorzubereiten, ist wahr $\mathrm{zu}_{\mathrm{e}}$ dann, wenn

(5a) $S_{\text {ingr }}$ das K unmittelbar nach $t_{e}$ impliziert; und

(5b) $S_{\text {ingr }}$ die Negation von $K,-K$, unmittelbar vor te präsupponiert. 
Parallel die Wahrheitsbedingungen für die restlichen zwei Subklassen von PV. Ein Satz mit egressivem Phasenverb $\mathrm{S}_{\mathrm{egr}}$, z.B. Das Kind hörte zu brüllen auf, ist wahr zu te dann, wenn

(6a) $S_{\text {egr }}$ das -K unmittelbar nach $t_{e}$ impliziert; und

(6b) $\mathrm{S}_{\text {egr }}$ das $\mathrm{K}$ unmittelbar vor $\mathrm{t}_{\mathrm{e}}$ präsupponiert.

Ein Satz mit kontinuativem Phasenverb $\mathrm{S}_{\mathrm{kont}}$, z.B. Das Kind fuhr zu brüllen fort, ist wahr zu te dann, wenn

(7a) $S_{\text {kont }}$ das K unmittelbar nach $t_{e}$ impliziert; und

(7b) $S_{\text {kont }}$ das $K$ unmittelbar vor $t_{e}$ präsupponiert.

Aus dieser präsuppositionshaltigen Semantik von Phasenverben kann eine präsuppositionslose, mehr kanonisierte Version der Phasenverbsemantik hergestellt werden (vgl. Engerer 2002: 60f.), auf die ich aber hier nicht eingehen kann. Bei dem nun folgenden Durchgang von Dowtys Word Meaning and Montague Grammar (Dowty 1979), mit dem ich abschließen will, wird diese Semantik immer wieder aufscheinen.

\section{ZUSTANDSWECHSEL BEI DOWTY}

In Dowtys bekanntem Werk Word Meaning and Montague Grammar (Dowty 1979) liegen die Wurzeln der Einfachen Phasensemantik begraben und es kann daher zur Beschreibung der Phasenverbsemantik - und zu einem besseren Verständnis der mit ihr verbundenen Probleme - beitragen. In diesem Klassiker, der explizit der Zeitsemantik von Verben gewidmet ist, werden Phasenverben immer wieder angesprochen, allerdings ohne sie als grammatisch relevante Gruppe mit eigenem Recht anzusehen; Dowtys Studie hat den dezidierten zeitgebundenen Hintergrund, generative Semantik und Montague-Grammatik einander näher zu bringen, und verfolgt daher in seinem Hauptimpetus ganz andere 


\section{PHASEN, ZUSTANDSWECHSEL UND DIE EINFACHE PHASENSEMANTIK}

Erklärungsziele als die einer Phasensemantik. Bei der Lesung muss also von solcherart motivierten Argumentationen abstrahiert werden.

Dowtys Re-Analyse der vier Vendlerschen Verbklassen Activities, States, Accomplishments und Achievements (s. die Diskussion in Dowty 1979: 51ff.; vgl. auch Vendler 1957) erklärt eine im Grunde aktionsartliche Klassifikation auf lexikalisch-dekompositionelle Weise, indem die Bedeutung verbaler Ausdrücke in primitive atomare Zustandsprädikate plus einer Reihe Satzoperatoren und logischer Partikeln zerlegt wird. Grundlegend für sein „Aspektkalkül“ ist also der Begriff des Zustandes („stative“) sowie des Zustandswechsels, aus denen mit Hilfe einfacher logischer Mittel alle anderen verbalen Klassen, insbesondere Accomplishments und Achievements, hergeleitet werden können. Dowtys Argumente für den primitiven Status von Zustandsprädikaten sind die Einfachheit ihrer semantischen Repräsentation in einer nur durch Zeiten angereicherten Prädikatenlogik sowie die Tatsache, dass ihre modelltheoretische Interpretation durchschaubar und unmittelbar plausibel ist (Dowty 1979: 74). Diese Feststellungen treffen, wenn ich Dowty richtig lese, neben Zuständen auch für Prozesse („,activities“ oder Aktivitäten) zu, also beides Verbalgruppen, die nicht Ereignisse im engeren Sinne denotieren (siehe den Eintrag "Ereignissemantik" im Lexikon der Sprachwissenschaft, vgl. Bußmann 2002: 196f.).

Komplexer stellt sich für Dowty die semantische Beschreibung von telischen Accomplishments und Achievements dar, welche Ereignisse im engeren Sinne bezeichnen (vgl. Dowty 1979: 74 sowie Bußmann 2002: 197). Ereignisse dieser Art sind nicht, wie Zustände und Prozesse, wahr oder falsch in einem bestimmten Zeitabschnitt oder an einem bestimmten Zeitpunkt, sie finden statt in der Zeit, wie Dowty (1979: 74) es ausdrückt. Dowty zieht hier Georg von Wrights Vorschlag heran, nach dem jedes Ereignis als Zustandswechsel zwischen zwei Zuständen definiert werden kann, wobei einer der Zustände immer die Negation des anderen ist (Dowty 1979: 74f.). Neben Propositionen und der Negation ist dann repräsentationstechnisch nur noch ein Symbol für die zeitliche Vor-Nach-Relation vonnöten, um eine griffige Darstellung für Zustandswechsel zu haben (bei von Wright der zweistellige Operator T) (Dowty 1979: 75): 
(8a) -pTp ,Zustand p tritt ein'

(8b) pT-p ,Zustand p ist zu Ende'

(8c) pTp ,Zustand p verbleibt, herrscht weiter'

(8d) -pT-p ,Zustand -p verbleibt' oder ,Zustand p versäumt einzutreten'

Die Parallele zur Phasenverbsemantik ist in diesem Schema unmittelbar klar, wir haben in von Wrights T-Kalkül, wie es auch genannt wird, zusätzlich die Präzisierung, dass die zeitliche Vor- und Nachproposition Zustände denotieren. Eine solche Beschränkung ist in der Phasenverbsemantik nicht enthalten, welche dafür mit Präsuppositionen und Implikationen hantiert.

Ich möchte auch darauf hinweisen, dass die Miteinbeziehung der Konstellationen mit gleich bleibenden Vorzeichen vor und nach $\mathrm{T}$ nicht mit Dowtys beziehungsweise von Wrights Charakterisierung von Ereignissen als Zustandswechsel zwischen Zuständen mit wechselnden Negationszeichen in Einklang steht. pTp und -pT-p sind keine Zustandswechsel und repräsentieren die Gruppe der Kontinuativa in der Standardphasensemantik. Ich habe den grammatisch speziellen Status der Kontinuative mehrmals hervorgehoben, hier könnten wir eine Teilbegründung vorfinden: Während Ingressive (-pTp) und Egressive (pT-p) als richtige Ereignisse durch einen regulären Zustandswechsel gekennzeichnet sind (formal gekennzeichnet durch einen Wechsel des Negationsoperators), denotieren Kontinuative keine Zustandswechsel, und bezeichnen deshalb auch keine Ereignisse. Folgerichtig klassifiziert Dowty die englischen kontinuativen Verben keep und continue an anderer Stelle dann auch als Activities, Prozesse (Dowty 1979: 68).

Dowty konstatiert nun eine Analogie zwischen von Wrights Logik der Zustandswechsel und der Gruppe von atomaren Prädikaten um BECOME, die von den frühen generativen Semantikern wie Lakoff und McCawley als ziemlich sichere Kandidaten für die dekompositionelle Analyse angesehen wurden. Als Beispiele führt Dowty folgende Sätze an (Dowty 1979: 75):

(9a) The soup cooled. - The soup is cool.

(9b) The door closed. - The door is closed. 


\section{PHASEN, ZUSTANDSWECHSEL UND DIE EINFACHE PHASENSEMANTIK}

Beide Sätze haben identische Relationen zu ihren prädikativen Gegenstücken mit is, repräsentiert durch das abstrakte Verb BECOME. Der erste Satz, The soup cooled, ist nur dann wahr, wie Dowty ausführt (Dowty 1979: 75), wenn das Nicht-Kühl-Sein der Suppe zum Referenzzeitpunkt des Satzes mit dem Zustand des Kühl-Seins (The soup is cool) erstattet wird. Folgt man Dowty, gilt diese Semantik für alle Sätze, deren Repräsentation das atomare Prädikat BECOME enthält.

Hierzu ist zu sagen, dass diese Analyse der Semantik des englischen Verbs to cool nicht auf das deutsche Pendant abkühlen übertragen werden darf. Während das englische Präteritum cooled, das in paradigmatischer Opposition zur Continuous Form was cooling steht, den vollendeten Aspekt signalisiert und damit Dowtys Explikation, dass der Zustand des Kühl-Seins eingetreten ist und den vorhergehenden Zustand erstattet, legitimiert und für das Englische als gültig erweist (vgl. Sasse 2006), fehlt im Deutschen diese Aspektopposition mit der Folge, dass aus Sätzen wie Die Suppe kühlte ab nicht geschlossen werden darf, dass die Suppe (schon) abgekühlt/kühl ist. Sie kann (in der Vergangenheit) durchaus abkühlen, aber trotzdem (noch) brennend heiß sein. Dies ist ein systematischer, den Aspektmarkierungen geschuldeter Unterschied zwischen dem Englischen und dem Deutschen, der in der Anwendung auf Deutsche berücksichtigt werden muss.

Dowty kommt zum Schluss, dass BECOME-Sätze, sowie deren Inverse END und das dritte atomare Prädikat REMAIN innerhalb von von Wrights Logik des Zustandwechsels beschrieben werden können (Dowty 1979: 75):

(10a) BECOME $(\mathrm{p})=$ def $-\mathrm{pTp}$

(10b) $\quad$ END $(p)=\operatorname{def}_{\text {pT }}$ p

(10c) $\operatorname{REMAIN~}(\mathrm{p})=$ def $\mathrm{pTp}$

Seine Analyse, in welcher die kontinuative Variante von REMAIN -pT-p offenbar heraus gefallen ist, deutet an, dass Phasenverbbedeutungen mithilfe der oben genannten atomaren Prädikate wiedergegeben werden können. Diesem schwachen Postulat steht die starke Konsequenz gegenüber, die besagt, dass 
atomare Phasenprädikate des Typs BECOME, END und REMAIN systematisch in die Bedeutung vieler anderer Verben eingehen (mindestens aller telischen Verben), Phasenprädikate damit keineswegs auf die Formative von Phasenverben angewiesen sind. Dies nachzuweisen ist, im Fahrwasser der generativen Semantik, in der sich Dowty bewegt, auch sein Anliegen. Obwohl es unabhängige Ansätze zum starken Postulat von Phasenprädikaten gibt, ${ }^{\bullet}$ möchte ich hier die schwache Version, also die Semantik von Phasenverben formuliert mit atomaren Prädikaten, verfolgen.

Dass Phasenverben und deren Synonyme korrekt mit atomaren Phasenprädikaten beschrieben werden können und sollten, wird von Dowty explizit gefordert (vgl. Dowty 1979: 75). Anfang und Ende von NichtEreignissen, also Zuständen und Prozessen, können zutreffend mit Phasenverben markiert werden, denen die von Wright'sche Theorie von Zustandswechseln zugrunde liegt:

(11a) Es begann zu regnen. (Zustand, BECOME)

(11b) John hörte auf zu laufen. (Prozess, END)

(11c) Harry aß sein Eis einfach weiter. (Prozess, REMAIN)

Um die atomaren Phasenprädikate mit ihrer am T-Kalkül angelehnten Semantik formal weiterverarbeiten zu können, interpretiert Dowty BECOME, END und REMAIN als Satzoperatoren, auf denen die Wahrheitsbedingungen direkt definiert werden können (Dowty 1979: 76; vgl. auch Engerer 2000a: 106ff.). Ist $\varphi$ eine Formel und $t$ irgendein Zeitpunkt, dann gilt:

(12a) BECOME $\varphi$ ist zu t wahr genau dann, wenn $\varphi$ zu t wahr ist und falsch zu $\mathrm{t}-1$.

(12b) END $\varphi$ ist zu t wahr genau dann, wenn $\varphi$ zu t falsch ist und wahr zu t-1.

(12c) REMAIN $\varphi$ ist zu t wahr genau dann, wenn $\varphi$ zu t wahr ist und wahr zu t1.

Es scheint mir der richtige Weg zu sein, Phasenprädikate als Satzoperatoren anzusehen, auch die einfache Semantik von Phasenverben läuft auf diese Lösung hinaus. Allerdings gibt es einen wichtigen Unterschied zwischen obiger 


\section{PHASEN, ZUSTANDSWECHSEL UND DIE EINFACHE PHASENSEMANTIK}

Formulierung der Wahrheitsbedingungen und denen von ePS, der darin besteht, dass die Standardsemantik (mindestens) mit drei Zeitpunkten, $t, t-1$ und $t+1$ arbeitet, Dowtys Version hingegen nur mit den beiden Punkten $t$ und $t-1$. Die letztere Lösung ist ganz klar vorzuziehen, denn sie erlaubt es, die Wahrheit eines Satzes mit Phasenoperator PO, PO $\varphi$, zu demselben Zeitpunkt t zu evaluieren wie die eingebettete Formel $\varphi$. In der Standardversion hängt der SatzEvaluierungszeitpunkt $t$ sozusagen in der Luft, da er auf der rechten Seite der Wahrheitsbedingungen nicht mehr vorkommt, denn nur Punkte vor oder nach $\mathrm{t}$ gehen in die Wahrheitsbedingungen ein.

Ein weiterer Vorteil der obigen Formulierung der Wahrheitsbedingungen liegt darin, dass sich die Wahrheitsbedingungen nicht auf einen Zeitpunkt nach dem Evaluierungszeitpunkt, also relativ von $t$ gesehen in der Zukunft, beziehen $(t+1)$. Dies macht Sätze im Präsens, wo t mit dem Sprechzeitpunkt zusammenfällt, unmittelbar interpretierbar, vgl.

Es beginnt zu regnen.

Dieser Satz ist nach der Dowty'schen Formulierung der Semantik des BECOMEOperators $\mathrm{zu}$ t, dem Sprechzeitpunkt, wahr genau dann, wenn es zum Sprechzeitpunkt regnet, und zum Zeitpunkt t-1, dem direkten Vorgänger von $t$ (wir nehmen an, Zeit sei ein diskretes Phänomen), es nicht geregnet hat. In der Standardversion der Phasenverbsemantik wäre ein solcher Satz nicht so ohne weiteres $\mathrm{zu}$ interpretieren.

Wie bereits gesagt, betrachtet Dowty Zustände („states“) als primitive Größen in der logischen Struktur von Verbbedeutungen; Aktivitäten („activities“) sind durch das atomare Prädikat DO abgeleitete Zustände, wobei DO eine Relation zwischen einem Individuum I und einem Zustandsprädikat P beschreibt und I referenzgleich ist mit dem ersten Argument von P (Dowty 1979: 124).

Achievements sind allesamt in dem Sinne ingressiv, da sie definitionsgemäß das Zustandswechselprädikat BECOME enthalten; BECOME ist einstellig und bettet Zustandsprädikate, also semantische Atome, ein, jedoch auch komplexere Ausdrücke wie Aktivitäten (DO-Derivate) und Accomplishments, welche durch das Prädikat CAUSE gekennzeichnet sind. In Bezug auf die beiden letzten Fälle 
komplexer eingebetteter Ausdrücke, die von Dowty explizit „Inchoation of Activity/Accomplishments“, also Ingressiva, genannt werden (Dowty 1979: 124), kann festgehalten werden, dass sie im Englischen nicht als Verbform lexikalisiert sind, jedoch periphrastischen Konstruktionen mit Phasenverb entsprechen; das Deutsche hingegen hat im Falle eingebetteter Aktivitäten das morphologische Mittel der Präfigierung mit los-. Vgl.

(14a) John began to walk (eingebettete Aktivität) vs. dt. John ging los

(14b) John began to build a house (eingebettetes Accomplishment)

Accomplishments, die letzte Vendlersche Verbgruppe, zeichnen sich durch das kausale Prädikat CAUSE aus, eine Art zweistelliger Satzverknüpfer ([ $\varphi$ CAUSE $\psi]$, vgl. Dowty 1979: 91ff.), wobei $\varphi$ und $\psi$ Achievements oder Aktivitäten sein können und die jeweilige Kombination und syntaktische Struktur der jeweiligen logischen Form es erlaubt, empirisch relevante Untertypen der AccomplishmentKlasse zu unterscheiden (vgl. Dowty 1979: 110ff., 124f.). Allgemein gesagt, involviert die Bedeutung von Accomplishment-Verben immer das Eintreten eines bestimmten Zustandes (Dowty 1979: 133).

\section{SCHLUSS}

Die zweite Phase der Erforschung von Phasenverben, im Sinne der eingangs vorgelegten Periodisierung, war also auf die semantischen Eigenschaften dieser Verbgruppe gerichtet, und bereits angelegt in der Form, in der sie in einer modernen Fassung in der ePS, Einfachen Phasensemantik, vorliegen. Dowty kann in dieser Hinsicht als Versuch einer Generalisierung von Phasenbedeutungen im Gesamtlexikon einer Sprache gelesen werden. Dieser Aspekt von universalen lexikonstrukturierenden Phasenbedeutungen ist, manchmal mehr und manchmal weniger explizit, Gemeingut in den Forschungsansätzen der 80er und 90er Jahre geworden. Dies zu zeigen ist kommenden Arbeiten vorbehalten. 


\section{PHASEN, ZUSTANDSWECHSEL UND DIE EINFACHE PHASENSEMANTIK}

\section{LITERATUR}

Althaus, Hans Peter, Helmut Henne, Herbert Ernst Wiegand (Hgg.) (1980), Lexikon der germanistischen Linguistik, Tübingen: Niemeyer.

Ballmer, Thomas T., Waltraud Brennenstuhl (1986), Deutsche Verben. Eine sprachanalytische Untersuchung des deutschen Verbwortschatzes, Tübingen: Gunter Narr.

Bennett, Michael, Barbara H. Partee (2004), "Toward the Logic of Tense and Aspect in English", in: Partee, Barbara H. (2004), Compositionality in Formal Semantics, Malden: Blackwell, pp 59-109.

Bierwisch, Manfred (1983), "Semantische und konzeptuelle Repräsentation lexikalischer Einheiten“, in Ruzicka, Rudolf, Wolfgang Motsch (Hgg.) (1983), Untersuchungen zur Semantik, Berlin: Akademie-Verlag, pp. 61-99.

Brown, Keith (ed.) (2006), Encyclopedia of Language \& Linguistics, Oxford: Elsevier.

Bußmann, Hadumod (2002), Lexikon der Sprachwissenschaft, Stuttgart: Kröner.

Cann, Ronnie (1993), Formal Semantics: an Introduction, Cambridge: Cambridge University Press.

Chierchia, Gennaro, Sally McConnell-Ginet (1990), Meaning and Grammar: an Introduction to Semantics, Cambridge/MA: MIT Press.

Cresswell, M. J. (1985), Adverbial Modification: Interval Semantics and its Rivals, Dordrecht, Boston, Lancaster, Tokyo: D. Reidel.

Dowty, David R. (1979), Word meaning and Montague grammar. The semantics of verbs and times in generative semantics and in Montague's PTQ Dordrecht: D. Reidel.

Dowty, David R. (1977), "Toward a semantic analysis of verb aspect and the English 'imperfective' progressive", Linguistics and Philosophy, 1 (1977), pp. 45-77.

Engerer, Volkmar (2008), „Vorschlag zu einer Systematik der grammatischen Beschreibung von Phasenverben“, in: Pittner, Karin (Hg.) (2008), Vorträge der 16. Jahrestagung der Gesellschaft für Sprache und Sprachen (Gesus e.V.), München: Lincom Europa, pp. 173 - 182 (= Beiträge zu Sprache und Sprachen 6, Edition Linguistik, 66).

Engerer, Volkmar (2007), "Faseverbet begynde: dets syntaks og semantik i dansk", i: Widell, Peter, Ulf Dalvad Berthelsen (red.) (2007), 11. Møde om Udforskningen af Dansk Sprog. Aarhus Universitet 12.-13. oktober 2006, Århus: Nordisk Institut, pp. 91-100.

Engerer, Volkmar (2003), "Indledning til "Tid i sprog": Sproglige tidskategorier. Hvad lingvister (måske) kan blive enige om", Tidsskrift for Sprogforskning, 1 (2003), pp. 5-20.

Engerer, Volkmar (2002), "Faseverbernes tidsbetingelse. Grundtræk af den semantiske beskrivelse af faseverbkonstruktioner", Hermes, 29 (2002), pp. 5371. 
Engerer, Volkmar (2001), "Phasenverben brauchen Zeit: Die zeitsemantische Verankerung von Satzgliedern", Studia Germanica Universitatis Vesprimiensis, 5 (2001), pp. 5-20.

Engerer, Volkmar (2000a), "Phasenverbsemantik", in: Dorfmüller-Karpusa, Käthi, Ekaterini Vretta-Panidou (Hgg.) (2000), Thessaloniker Interkulturelle Analysen. Akten des 33. Linguistischen Kolloquiums in Thessaloniki 1998, Frankfurt/M., Berlin, Bern, Bruxelles, New York, Oxford, Wien: Peter Lang, pp. 101-110.

Engerer, Volkmar (2000b), "Was lässt sich im Deutschen aufhören und beenden? Aspekt, Aktionsart und nominale Komplementation intervallabschlussdenotierender Phasenverben", Odense Working Papers in Language and Communication, 19 (2000), pp. 171-186.

Engerer, Volkmar (1999), "Phasenverbkomplemente", Studia Germanica Universitatis Vesprimiensis, 3 (1999), pp. 145-159.

Fabricius-Hansen, Cathrine (1975), Transformative, intransformative und kursive Verben, Tübingen: Max Niemeyer.

Galton, A. (2006), "Temporal Logic", in: Brown, Keith (ed.) (2006), Encyclopedia of Language \& Linguistics, Oxford: Elsevier, pp. 563-566.

Givon, Talmy (1973), "Forward Implications, Backward Presuppositions, and the Time Axis of Verbs", in: Kimball, John P. (ed.) (1973), Syntax and Semantics, New York, London: Seminar Press, pp.29-50.

Helbig, Gerhard, Joachim Buscha (1984), Deutsche Grammatik. Ein Handbuch für den Ausländerunterricht, Leipzig: Verlag Enzyklopädie.

Jun, J. S. (2006) "Lexical Conceptual Structure", in: Brown, Keith (ed.) (2006), Encyclopedia of Language \& Linguistics, Oxford: Elsevier, pp. 69-77.

Lohnstein, Horst (1996), Formale Semantik und natürliche Sprache. Einführendes Lehrbuch, Opladen: Westdeutscher Verlag.

Mulisch, Herbert (Hg.) (1985), Morphologie. Die russische Sprache der Gegenwart, Bd. 2, Leipzig: Verlag Enzyklopädie.

Partee, Barbara H. (2004), Compositionality in formal semantics. Selected papers, Malden: Blackwell.

Pustejovsky, James (1995), The Generative Lexicon, Cambridge/MA: MIT Press.

Reichenbach, Hans (1947), Elements of symbolic logic, New York: Macmillan.

Sasse, H. -J. (2006), "Aspect and Aktionsart", in: Brown, Keith (ed.) (2006), Encyclopedia of Language \& Linguistic, Oxford: Elsevier, pp. 535-538.

Stechow, Arnim von, Wolfgang Sternefeld (1988), Bausteine syntaktischen Wissens. Ein Lehrbuch der generativen Grammatik, Opladen: Westdeutscher Verlag.

Trask, R. L. (1993), A dictionary of grammatical terms in linguistics, London: Routledge.

Vendler, Zeno (1957), "Verbs and Times", Philosophical Review, 66 (1957), pp.143-160.

Zifonun, Gisela, Ludger Hoffmann, Bruno Strecker (1997), Grammatik der deutschen Sprache, Berlin: de Gruyter. 
PHASEN, ZUSTANDSWECHSEL UND DIE EINFACHE PHASENSEMANTIK

\section{ANMERKUNG}

- Vgl. die umfangreiche Untersuchung zum deutschen Verbwortschatz in Ballmer und Brennenstuhl (Ballmer/Brennenstuhl 1986), wo Phasenprädikate (dort „Phasenverben“ genannt) im Sinne der dekompositionellen Hypothese den deutschen Verbwortschatz im Ganzen strukturieren. Vgl. die Diskussion dieses Ansatzes in Engerer (2000a) und die dort vertretene Konzeption eines Phasenverboperators, der atomaren Phasenprädikaten sehr ähnlich ist. 